\title{
Individual differences in attitude toward robots predict behavior in human-robot interaction
}

\author{
Nina-Alisa Hinz ${ }^{10000-0002-7830-1482]}$, Francesca Ciardo ${ }^{2}$ [0000-0002-2530-3089] and Agnieszka \\ Wykowska ${ }^{2}$ [0000-0003-3323-7357] \\ ${ }^{1}$ General and Experimental Psychology, Ludwig-Maximilians-University, Geschwister-Scholl- \\ Platz 1, 80539 München, Germany \\ ${ }^{2}$ Social Cognition in Human-Robot Interaction, Italian Institute of Technology, Via Enrico \\ Melen, 83, 16152 Genova, Italy \\ francesca.ciardodit.it
}

\begin{abstract}
Humans are influenced by the presence of other social agents, sometimes performing better, sometimes performing worse than alone. Humans are also affected by how they perceive the social agent. The present study investigated whether individual differences in the attitude toward robots can predict human behavior in Human-Robot Interaction (HRI). Therefore, adult participants played a game with the Cozmo robot (Anki Inc., San Francisco), in which their task was to stop a balloon from exploding. In individual trials, only the participants could stop the balloon inflating, while in joint trials also Cozmo could stop it. Results showed that in joint trials, the balloon exploded less often than in individual trials. However participants stopped the balloon earlier in joint than in individual trials, although this was less beneficial for them. This effect of Cozmo joining the game, nevertheless, was influenced by the negative attitude of the participants toward robots. The more negative they were, the less their behavior was influenced by the presence of the robot. This suggests that robots can influence human behavior, although this influence is modulated by the attitude toward the robot.
\end{abstract}

Keywords: Individual differences, Attitude toward robots, Human-Robot Interaction.

\section{Introduction}

Robotic agents are already present in many aspects of our everyday-life, whether they greet us at the airport [1], assist in elderly care [2] or work side-by-side with human employers in manufacturing [3]; and wider applications are probably going to emerge. Therefore, humans face the need to act more and more often in the presence of robots or to interact with them. In situations with social or work-related responsibility, it appears important to know how people behave in the presence of robots and how this eventually diverges from individual situations.

Although evidence showed that the presence of social agents in the environment may improve performance, it may also create difficulties $[4,5]$. For instance, in human- 
human interaction, a well-known phenomenon is social facilitation, i.e. the fact that the presence of another human can enhance performance [4]. However, this effect is dependent on the nature of the task [6]. For complex and difficult tasks, for example, the presence of a co-agent can lead to social inhibition, i.e. deteriorated performance [5]. Similar effects have been demonstrated with artificial agents [7, 8]. As has been demonstrated before in other aspects of social cognition [9], embodiment seems to affect the social presence effect, as presence induced by images of social agents did not affect performance [10]. These studies, however, have used fairly simple tasks, such as arithmetic operations, that do not accurately resemble the complexity that characterizes practical applications of robots. Indeed, most of the applications in which a human has to interact with a robot rather deal with uncertainty and risky contexts.

Another crucial aspect to examine in HRI is how individual differences in robot perception translate to differences in behavior $[11,12]$. Indeed, individual differences, such as personality traits or attitudes, have been found to affect the perception and acceptance of robots in social situations [13-16]. For example, higher levels of neuroticism seem to predict the preference of more machine-like robots [13]. Similarly, differences in assigning human-like characteristics to non-human agents lead to differences in the trustworthiness assigned to a robot [14]. A full range of individual differences, like anxiety, perfectionism or religious fundamentalism, have been proposed to explain feelings of eeriness towards the robot [15]. Differences in trustworthiness perception of a robot have been demonstrated to predict behavior in a human-robot team [16-17].

\subsection{Aim of the study}

Our study aimed at investigating whether human behavior is influenced by the presence of a robotic agent in a task necessitating risk-taking, and how this influence is moderated by individual differences in the attitude towards robots. Therefore, we asked participants to perform a game in which they had to stop a balloon from exploding alone or playing with the Cozmo robot (Anki Inc., San Francisco). We used a non-anthropomorphic robot in order to avoid that differences in assigning human-likeness might affeet trustworthiness toward the robot [14]. We expected Cozmo to improve the performance of participants in the task due to social facilitation effects as a function of individual differences.

\section{Methods}

\subsection{Participants}

Thirty-two healthy adults participated in this study. Data of one participant were not analyzed because the robot crashed during the experimental session. The remaining sample consisted of thirty-one participants (12 male, 1 left-handed, age range: 19 -44 years, $\mathrm{M}=23.85, \mathrm{SD}=4.81$ ). All had normal or corrected- to-normal vision. Partici- 
pants provided informed written consent before participation, received financial reimbursement and were debriefed after the experiment. The study was approved by the local ethical committee (Comitato Etico Regione Liguria).

\subsection{Apparatus and Materials}

Participants were seated in front of a desk on which lay a computer screen (22 inches diagonal, 1366x768 pixels resolution, $59 \mathrm{~Hz}$ refresh rate). The Cozmo robot was placed directly in front of them (see Fig. 1). For both the participant and the Cozmo robot, the response device was one of the Cozmo cubes on top of which an in-house-built onekey-keyboard was mounted.

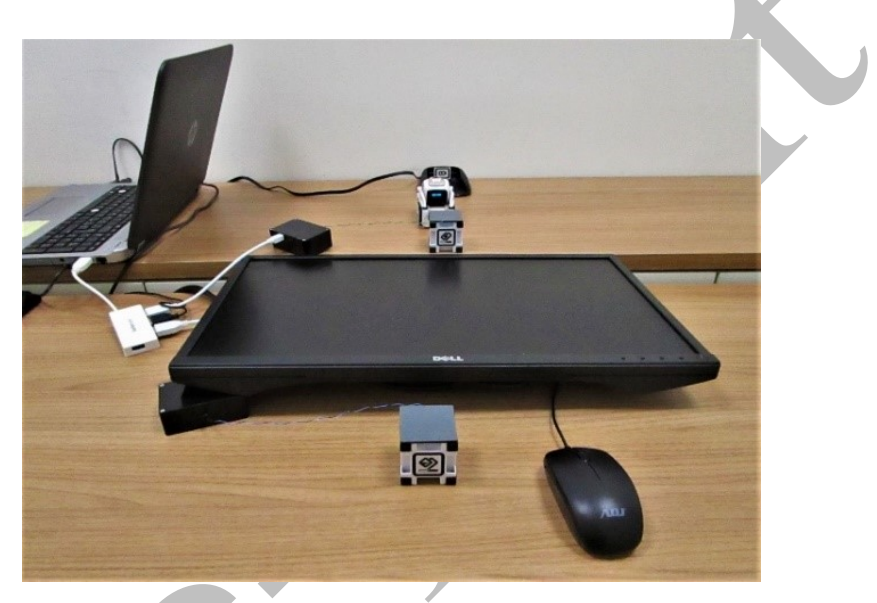

Fig. 1. Experimental Setup.

\section{The Cozmo robot}

The Cozmo robot (Anki Inc., San Francisco) is a commercial robot designed for educational purposes. It consists of a horizontally moveable head with an LED screen on which eyes are displayed, four wheels, three LEDs on the back and a horizontally moveable lift. Cozmo is controlled by an application compatible with iOS and Android. A Python 3.6-based Software development kit (SDK) can be used to program Cozmo.

During the experiment a mobile Android device with the Cozmo application in "SDK mode" was used to control Cozmo, connected to a laptop through the Android Debug Bridge (adb) as described in [18]. Commands were sent to the Cozmo application by using OpenSesame Version 3.1.9 [19], running on Python 3.6.

\section{Questionnaires}

To assess the participant's attitude towards robots, before the experiment we administered three different questionnaires. All of the questionnaires were presented on a computer screen, using OpenSesame Version 3.1.9 [19] and responded to with a standard computer mouse. The questionnaires were: 
- the Frankenstein Syndrome Questionnaire (FSQ; [20]) is composed of thirty items, measuring the "Frankenstein Syndrome", the fear of creating new entities. Four different subscales are evident in the questionnaire: "General Anxiety toward humanoid robots", "Apprehension toward social risks of humanoid robots", "Trustworthiness for developers of humanoid robots" and "Expectation for humanoid robots in daily life". The subscales have medium to good internal consistency, the questionnaire in general, however, demonstrated good reliability [21]. Higher scores stand for a more severe Frankenstein Syndrome, i.e. more negative attitude towards robots.

- the Negative Attitude Towards Robots Scale (NARS; [13]) consisting of fourteen items, organized in three different subscales: "Negative Attitudes toward Situations and Interactions with Robots", "Negative Attitudes toward Social Influence of Robots" and "Negative Attitudes toward Emotions in Interaction with Robots". The questionnaire demonstrated high internal consistency and validity. Higher scores stand for more negative attitude towards robots.

- the Robotic Social Attributes Scale (RoSAS; [22]) consisting of eighteen items in which participants are presented with an adjective (e.g. social) and have to express on a 9-point Likert scale how much they believe that the adjective can be used to describe a robot. Items are classified into three psychometrically validated subscales: "Warmth", "Competence" and "Discomfort". While higher scores on the subscales "Warmth" and "Competence" are related to a more positive attitude towards robots, higher scores on the "Discomfort" subscale signify a more negative attitude.

\subsection{Task and Trial Procedure}

The task was based on the Balloon Analogous Risk Task [23]. Participants played a game, in which they had to stop an inflating balloon before it exploded when reaching a pin on the top of the display. In every trial the participants would lose points from an initial amount of 4000 points, with the amount of lost points depending on when the balloon was stopped. The later the balloon was stopped, the less points were lost. The exact number of lost points per trial was randomly chosen from a range of points, depending on four different clusters of sizes at which the balloon was stopped (see Table 1). The maximal amount of points was lost when the balloon exploded. The goal of the game was to save the maximal amount of points; therefore the best strategy would have been to wait as long as possible before stopping the balloon. In $50 \%$ of trials, participants played alone (individual trials), while in the remaining trials also Cozmo was in charge to stop the balloon inflation (joint trials). Cozmo was programmed to act only in the $60 \%$ of joint trials. When Cozmo stopped the balloon, it would lose the respective amount of points, whereas the participant would lose no points. In case of an explosion both agents would lose the points. Cozmo always acted when approximately $90 \%$ of the inflation time were reached. In joint trials, the best strategy for the participant would have been to wait for an action of Cozmo and only react in trials, in which it did not, to prevent an explosion of the balloon. However, participants could not predict a priori when Cozmo would act.

At the beginning of each trial participants were told whether they were playing alone (individual trial) or with Cozmo (joint trial) with a text presented on the screen for 1000 
ms (see Fig. 2). During the first trial of each block Cozmo either went to sleep (transition from joint to individual trial) or woke up and approached the cube (transition from individual to joint trial). After the initial instruction about the type of trial, a sketchpad displaying "The trial is starting" was presented on the screen, so participants could prepare for the beginning of each trial. Then, a fixation point was presented for a random duration of 800-1000 ms (note that the fixation point duration was randomly set at the beginning of each trial and kept constant for all fixation points presented in the respective trials). After that, the images of a pin and of the balloon at its starting size were presented for $500 \mathrm{~ms}$. Following, the balloon started inflating. The inflation speed was variable across and within trials, in order to make the explosion time not predictable. In each block ten different inflation speeds were used. Additionally, during the inflation sequence, two grey circles were presented on the bottom right and top left corner. When a response was given one of the circles turned blue, indicating whose reaction was counted (with top left corner representing Cozmo and bottom right corner representing the participant). After a response was executed, the balloon was displayed in its final size for $1000 \mathrm{~ms}$. If the balloon exploded, an image representing the bursting event was presented. Then a fixation point was followed by a sketchpad $(2000 \mathrm{~ms})$ showing the amount of lost points.

Participants were explicitly instructed that their goal was to save as many points as possible to defeat previous participants and they would not be able to defeat Cozmo since it was playing in only half of trials. This was done to try to avoid that they would perceive the task as a competition between themselves and Cozmo.

The task consisted of 180 trials presented in 18 blocks of 10 trials each. The type of trials (individual or joint) was manipulated across blocks. The order of the blocks was randomly selected. A practice session of 6 trials was administered before the experiment. During the practice participants experienced one trial in which Cozmo was not reacting.

Table 1. Lost points depending on the balloon size at reaction. Balloon sizes were separated into four clusters. Depending on in which cluster the reaction was, a random number was drawn from the corresponding range.

\begin{tabular}{rr}
\multicolumn{2}{c}{ from the corresponding range. } \\
$\begin{array}{r}\text { Time of reaction } \\
\text { (percentage of inflation time) }\end{array}$ & Range of points lost \\
$\geq 50 \%$ & $1-15$ \\
$33-49 \%$ & $16-29$ \\
$17-33 \%$ & $31-45$ \\
$\leq 17 \%$ & $46-60$ \\
(explosion) $100 \%$ & $80-100$ \\
\hline
\end{tabular}




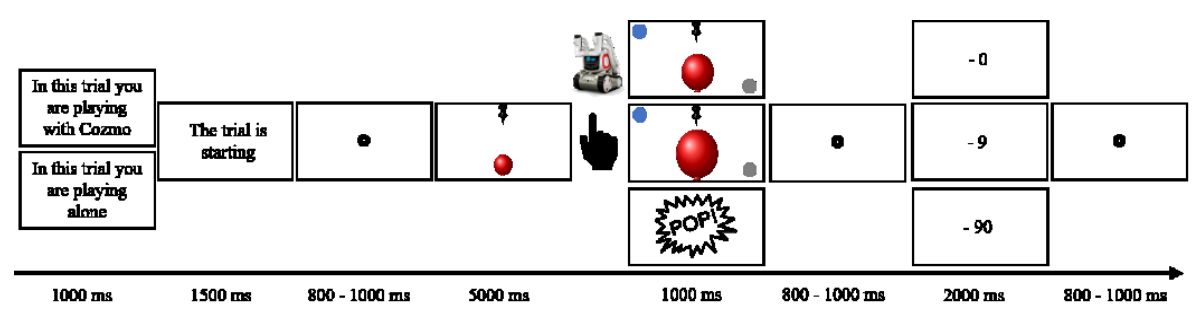

Fig. 2. Trial procedure. In joint trials Cozmo and the participant could stop the balloon from exploding. If Cozmo stopped the balloon the participant was not losing points. In individual trials only the participant could stop the balloon from exploding.

\subsection{Statistical Analysis}

Each trial was classified as a "Human" trial if the participant stopped the balloon and as a "Cozmo" trial if Cozmo stopped the balloon. If no one stopped the balloon and it exploded, the trial was classified as "No reaction". Performance was assessed by the amount of reactions in each trial type, reaction time (i.e. the amount of time in ms from the starting of the inflation until the balloon was stopped) and the points lost after the reaction. This behavioral data was analyzed using paired-sample t-tests. Ratings from the questionnaire subscales were summed up to a total score. Pearson correlations were conducted to examine the relationship between questionnaire (subscale and total) scores and the behavioral data. All analyses were performed using R Version 3.5.1 [24]. Plots were created using the ggplot2 package Version 3.0.0 [25].

\section{Results}

Participants successfully stopped the balloon in $85.4 \%(S D=7.7 \%$; see Error! Reference source not found.) of individual trials and $47.3 \%(S D=9.5 \%)$ of joint trials. In $42.1 \%(S D=6.7 \%)$ of joint trials they let Cozmo react. The balloon exploded in $14.6 \%(S D=7.7 \%)$ of individual trials, but only $10.6 \%(S D=4.4 \%)$ of joint trials. The number of explosions differed significantly between the two types of trials $\left(t_{29}=-3.55\right.$, $p<.001)$.

Given that Cozmo's actions were influencing the information contained in outcome and balloon size, only trials in which the participant successfully stopped the balloon (Human trials) were further analyzed. No difference was found between the points lost in each trial type (Joint: $M=9.05, S D=0.85$; Individual: $M=8.79, S D=0.74 ; t_{29}=$ 1.32, $p=.19$; see Fig. left). However, reaction times were different between the trial types $\left(t_{29}=-3.42, p<.001\right)$, showing faster performance in joint $(M=4078 \mathrm{~ms}, S D=$ $93 \mathrm{~ms})$ compared to individual trials $(M=4130 \mathrm{~ms}, S D=66 \mathrm{~ms}$; see Error! Reference source not found. left).

The analysis of correlations between questionnaires and performance (i.e. lost points, balloon sizes and reaction times) showed that the FSQ total score correlated negatively with the amount of lost points in the joint trials only (Joint: $r=.45, p<.01$; Individual: $r=.09, p=.62$; see Fig. right). No significant correlations were found 
between the amount of lost points and each FSQ-subscale score (all $p \mathrm{~s}>$.XX). Finally, the score on the discomfort scale of the RoSAS was positively correlated to reaction times in joint trials only (Joint: $r=.33, p=.07$; Individual: $r=.06, p=.76$; see Error! Reference source not found. right).

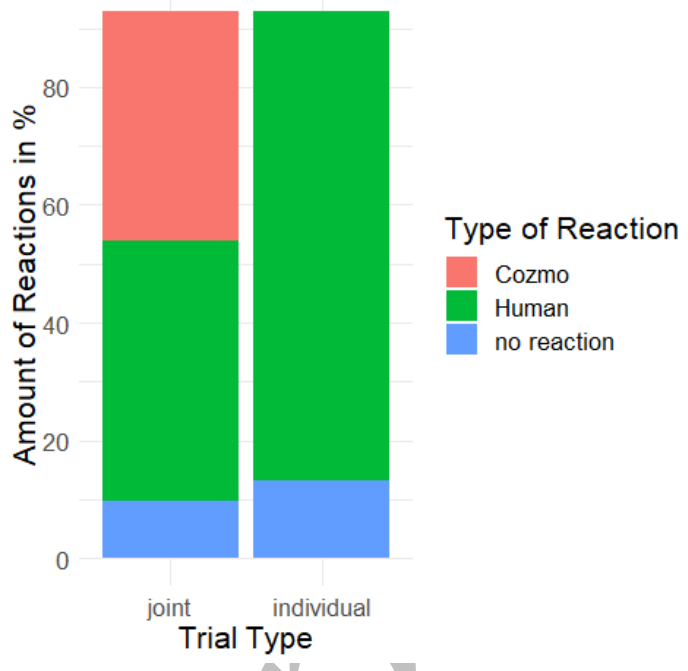

Fig. 3. Proportions of reactions in the two trial types. Cozmo was only acting in joint trials.

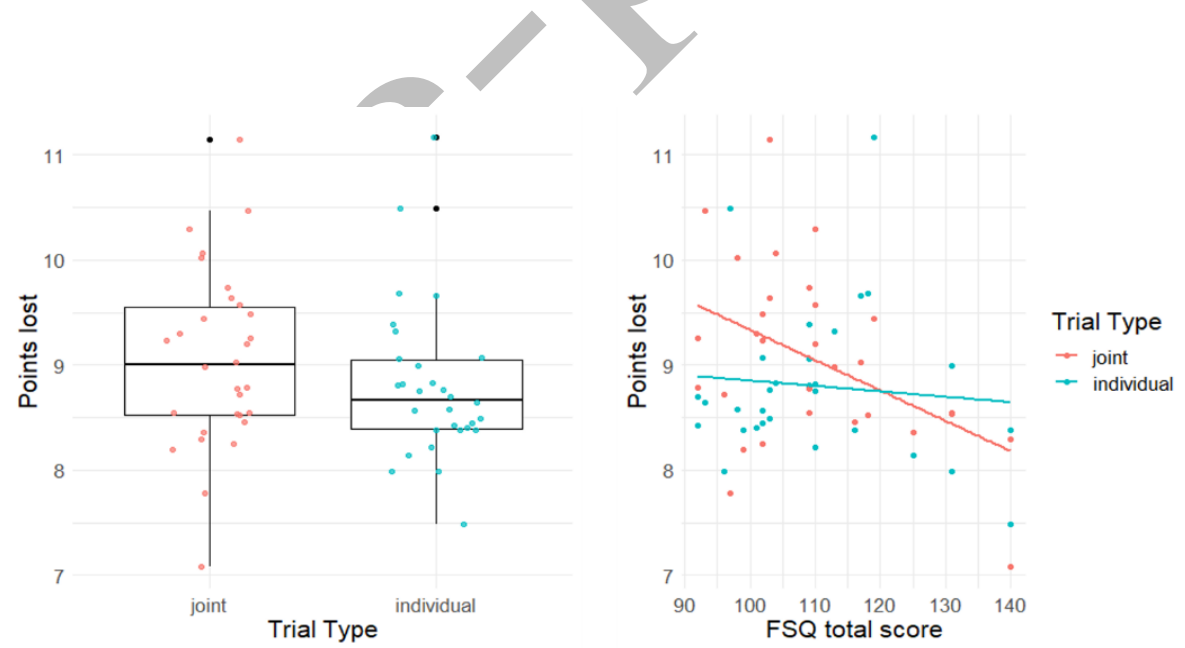

Fig. 4. Left panel: Average amount of lost points when participants successfully stopped the balloon as a function of trial type (individual vs. joint). Right panel: Correlations between the amount of lost points in Human trials and the FSQ total score. 

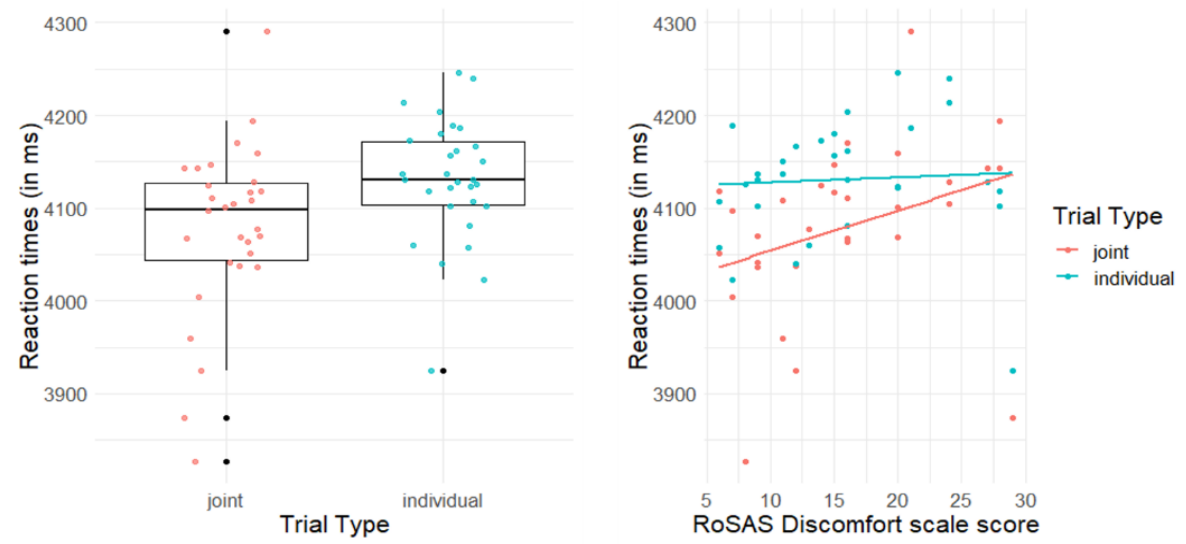

Fig. 5. Left panel: Average reaction times of trials in which the participants successfully stopped the balloon across trial type (individual vs. joint). Right panel: Correlations between reaction times in Human trials and the RoSAS Discomfort scale score.

\section{Discussion}

In the present study, we investigated how someone's behavior is influenced by the presence of a robotic agent and especially how this influence is moderated by individual differences in the attitude towards robots. Our results showed that when playing with Cozmo, participants overall performance in the game improved as indicated by a smaller number of explosions for joint than individual trials. This effect was clearly driven by Cozmo's actions. When only analyzing trials in which participants successfully stopped the balloon themselves (Human trials), results showed that participants stopped the balloon slightly earlier (as evident from faster reaction times) and at smaller sizes in the joint compared to the individual trials. Such a difference in the performance suggests that the presence of Cozmo triggered the action in joint trials, leading to a social facilitation effect exerted by the robot. Interestingly, the social facilitation effect occurred despite that the optimal strategy to lose as few points as possible was to react as late as possible (i.e. withholding the action). It should be noted that social facilitation was not observed in the number of lost points because the relation between stop size and actual feedback was not fully linear (see Table 1).

Our results showed that the effect exerted by the social presence of Cozmo varied as function of individual attitude towards robots, with higher scores on the FSQ questionnaire being associated with better performance (i.e. later balloon stops and fewer points

lost) in joint trials, and higher scores on the Discomfort subscale of the RoSAS leading to later balloon stops (i.e. slower reaction times) in joint trials. Together the correlation patterns suggest that the more negative someone is regarding robots, the less $\mathrm{s} / \mathrm{he}$ is influenced by the presence of the robot and the less s/he shows social facilitation effect. Such a result is in line with the hypothesis of Schellen and Wykowska [11], that negative attitudes towards robots might be a moderating factor for social presence effects. 
Future work should look at how the present effects translate to the use of other robots, especially humanoid robot. Humanoid robots could for example further increase social facilitation effects by inducing more social presence. Future studies should also account whether trustworthiness toward the robot may affect the decision to intervene earlier to prevent losing points.

\section{Conclusion}

Our results indicate that sharing a task with a robot apparently triggers the execution of action, even when it is not beneficial. This should be carefully considered when introducing robots in situations with social or work-related responsibility. In a similar vein, our results highlight the importance of carefully examining individual differences in the attitude towards robots, since they differently affect how people behave in the presence of a robotic agent.

\section{Acknowledgements}

This project has received funding from the European Research Council (ERC) under the European Union's Horizon 2020 research and innovation program (grant awarded to AW, titled "InStance: Intentional Stance for Social Attunement." G.A. No: ERC2016-StG-715058).

\section{$7 \quad$ References}

1. Flughafen München GmbH: Hi! I'm Josie Pepper, https://www.munichairport.com/hi-i-m-josie-pepper-3613413

2. Kachouie, R., Sedighadeli, S., Khosla, R., Chu, M.-T.: Socially Assistive Robots in Elderly Care. A Mixed-Method Systematic Literature Review. International Journal of Human-Computer Interaction 30, 369-393 (2014)

3. Innovative human-robot cooperation in BMW Group Production. Munich, Germany (2013)

4. Zajonc, R.B.: Social Facilitation. Science 149, 269-274 (1965)

5. Myers, D.G., DeWall, C.N.: Psychology (2018)

6. Ciardo, F., Wykowska, A.: Response Coordination Emerges in Cooperative but Not Competitive Joint Task. Frontiers in Psychology 9, 1919 (2018)

7. Park, S., Catrambone, R.: Social facilitation effects of virtual humans. Human factors 49, 1054-1060 (2007)

8. Riether, N., Hegel, F., Wrede, B., Horstmann, G.: Social facilitation with social robots? In: Proceedings of the Seventh Annual ACM/IEE International Conference on Human-Robot-Interaction, pp. 41-48 (2012)

9. Kompatsiari, K., Ciardo, F., Tikhanoff, V., Metta, G., Wykowska, A.: On the role of eye contact in gaze cueing. Scientific Reports 8, 17842 (2018) 
10. Hertz, N., Wiese, E.: Social Facilitation with Non-Human Agents. Possible or not? In: Proceedings of the Human Factors and Ergonomics Society Annual Meeting, 61, pp. 222-225 (2017)

11. Schellen, E., Wykowska, A.: Intentional Mindset Toward Robots-Open Questions and Methodological Challenges. Frontiers in Robotics and AI 5, 71 (2019)

12. Marchesi, S., Ghiglino, D., Ciardo, F., Perez-Osorio, J., Baykara, E., Wykowska, A.: Do We Adopt the Intentional Stance Toward Humanoid Robots? Frontiers in Psychology 10, 450 (2019)

13. Syrdal, D.S., Dautenhahn, K., Kony, K.L., Walters, M.L.: The Negative Attitudes towards Robots Scale and Reactions to Robot Behaviour in a Live Human-Robot Interaction Study. Adaptive and Emergent Behaviour and Complex Systems (2009)

14. Waytz, A., Cacioppo, J., Epley, N.: Who Sees Human? The Stability and Importance of Individual Differences in Anthropomorphism. Perspectives on Psychological Science 5, 219-232 (2010)

15. MacDorman, K.F., Entezari, S.O.: Individual differences predict sensitivity to the uncanny valley. IS 16, 141-172 (2015)

16. Rossi, S., Staffa, M., Bove, L., Capasso, R., \& Ercolano, G.: User's personality and activity influence on HRI comfortable distances. In: In International Conference on Social Robotics, pp. 167-177 (2017)

17. Rossi, S., Santangelo, G., Staffa, M., Varrasi, S., Conti, D., Di Nuovo, A.: Psychometric Evaluation Supported by a Social Robot. Personality Factors and Technology Acceptance. In: 2018 27th IEEE International Symposium on Robot and Human Interactive Communication (RO-MAN), pp. 802-807. IEEE (2018)

18. Anki Inc.: Android Debug Bridge (2016)

19. Mathôt, S., Schreij, D., Theeuwes, J.: OpenSesame. An open-source, graphical experiment builder for the social sciences. Behav Res 44, 314-324 (2012)

20. Nomura, T., Sugimoto, K., Syrdal, D.S., Dautenhahn, K.: Social acceptance of humanoid robots in Japan. A survey for development of the frankenstein syndrome questionnaire. In: $12^{\text {th }}$ IEEE-RAS International Conference on Humanoid Robots (Humanoids 2012), pp. 242-247 (2012)

21. Syrdal, D.S., Nomura, T., Dautenhahn, K.: The Frankenstein Syndrome Questionnaire-Results from a quantitative cross-cultural survey. In: Proceedings of the International Conference on Social Robotics, pp. 270-279 (2013)

22. Carpinella, C.M., Wyman, A.B., Perez, M.A., Stroessner, S.J.: The Robotic Social Attributes Scale (RoSAS). In:. Proceedings of the ACM/IEEE International Conference on Human-Robot Interaction, pp. 254-262 (2017)

23. Lejuez, C.W., Read, J.P., Kahler, C.W., Richards, J.B., Ramsey, S.E., Stuart, G.L., Strong, D.R., Brown, R.A.: Evaluation of a behavioral measure of risk taking. The Balloon Analogue Risk Task (BART). Journal of Experimental Psychology: Applied 8, 75-84 (2002)

24. R Core Team: R: A language and environment for statistical computing. Vienna (2018)

25. Wickham, H.: ggplot2: Elegant Graphics for Data Analysis. Springer-Verlag New York (2016) 